\title{
Spectral Analyses of Heart Rate Variability by Acceleration Plethysmography for Diagnostic Support of Migraine: Clinical Research
}

\author{
Shigeki Edahiro ${ }^{1,2,7}$, Takahiro Maruta ${ }^{3,4}$, Masako Negami ${ }^{5,8}$, Yumi Adachi4,6 and Hiroaki Yoshikawa ${ }^{1,4,7 *}$
}

${ }^{1}$ Division of Life Sciences, Graduate School of Natural Science and Technology, Kanazawa University, Kanazawa, Ishikawa, Japan

${ }^{2}$ Department of Pharmacology, Kanazawa Red Cross Hospital, Kanazawa, Ishikawa, Japan

${ }^{3}$ Neurological Center, Kanazawa-Nishi Hospital, Kanazawa, Ishikawa, Japan

${ }^{4}$ Health Service Center, Kanazawa University, Kanazawa, Ishikawa, Japan

${ }^{5}$ Health Service Center, Keiju Medical Center, Nanao, Ishikawa, Japan

${ }^{6}$ Division of School Education, Graduate School of Education, Kanazawa University, Kanazawa, Ishikawa, Japan

${ }^{7}$ Department of Neurology and Neurobiology of Aging, Kanazawa University Graduate School of Medical Science

${ }^{8}$ Department of Clinical Health Science, Tokai University School of Medicine, Japan

\begin{abstract}
It is suspected that the pathophysiology of migraine has a relation to the autonomic dysfunction. We studied to assess the autonomic function in migraine using the spectral analysis of heart rate variability (HRV) by accelerated plethysmography (APG). Five-hundred-twenty-four patients received the health check-ups in an affiliated hospital since September, 2011 to April, 2013. Eighty-three patients with headache and 38 controls were enrolled in this study. The patients were subdivided into 19 with migraine (19 female), 26 with tension-type headache (TTH) ( 7 male, 19 female) and 31 of other headaches using a questionnaire based on the International Classification of Headache Disorders 2nd Edition. Patients with migraine or tension-type headache underwent examinations using APG with an orthostatic load. Twenty-four controls without headache received same examinations. As a result, the standing/sitting ratio of spectral power of high-frequency (HF) in the migraine group was higher than those of TTH $(p=0.0277)$ and normal controls $(p=0.0104)$. The cut-off value of 1.058 could separate migraine from TTH with sensitivity of 0.632 and specificity of 0.846 . The results suggested that the parasympathetic activity accentuated by orthostatic load in patients with migraine, on the contrarily the parasympathetic activities diminished by orthostatic load in patients with TTH or normal controls. This study showed that spectral analysis of HRV by APG is a supportive tool to distinguish migraine from TTH.
\end{abstract}

Keywords: Migraine; Heart rate variability; Accelerated plethysmography; Parasympathetic nerve; Sympathetic nerve

\section{Introduction}

The majority of primary headaches are migraines and tensiontype headaches (TTH). Although knowledge about migraine has been accumulated, there are still unclear issues regarding its pathophysiology. The leading explanation of migraine involves the trigeminovascular theory $[1,2]$, which asserts its relationship with the trigeminal nerve and intracranial vessels.

Generally, in the course of migraine attacks, vasoactive substances trigger the cascades of migraine. Then, the processes of degranulation of mast cells, increased permeability of vessels and vasodilatation are induced, which cause nausea and vomiting in patients. After these processes, afferent transmissions are sensed as headache by patients, and the trigeminal nerves secrete neuropeptide at the nerve terminals.

Clinical observations that support these hypotheses are nasal congestion, teary eyes and lacrimation, which are also considered to be signs of a hyper-parasympathetic state [3]. These symptoms suggest that autonomic dysfunctions are taking place in patients with migraine $[4,5]$. Reports have been published regarding fluctuation of blood pressure or heart rate variability (HRV) in patients with migraine, but no general agreement has yet been obtained [6]. On the other hand, autonomic dysfunction is also suspected in the pathophysiology of TTH $[6,7]$.

Recently, HRV has been utilized in research in many fields [810]. HRV contains substantial information about hemodynamics and is utilized as an index of cardiovascular dynamics and autonomic functions $[8,10,11]$.

In this study, we analyzed the HRV obtained from an accelerated plethysmogram (APG) from patients with migraine or TTH to study the effect of the orthostatic alterations.

\section{Materials and Methods}

\section{Participants}

This study was approved by the ethics committees of Kanazawa University and Keiju Medical Center. Patient consent was obtained using a written consent form. We studied patients who underwent health check-ups at Keiju Medical Center from September 2011 to April 2013 (Figure 1).

We enrolled 524 patients received the health check-ups. If patients

*Corresponding author: Hiroaki Yoshikawa, Division of Life Sciences, Graduate School of Natural Science and Technology and Department of Neurology and Neurobiology of Aging, Kanazawa University, Kanazawa, Ishikawa, Japan; Tel: 81 76234 6806; E-mail: hiroaki@staff.kanazawa-u.ac.jp

Received April 22, 2015; Accepted April 28, 2015; Published April 29, 2015

Citation: Edahiro S, Maruta T, Negami M, Adachi Y, Yoshikawa H (2015) Spectral Analyses of Heart Rate Variability by Acceleration Plethysmography for Diagnostic Support of Migraine: Clinical Research. J Neurol Disord 3: 229. doi: 10.4172/23296895.1000229

Copyright: ( 2015 Edahiro S, et al. This is an open-access article distributed under the terms of the Creative Commons Attribution License, which permits unrestricted use, distribution, and reproduction in any medium, provided the original author and source are credited. 


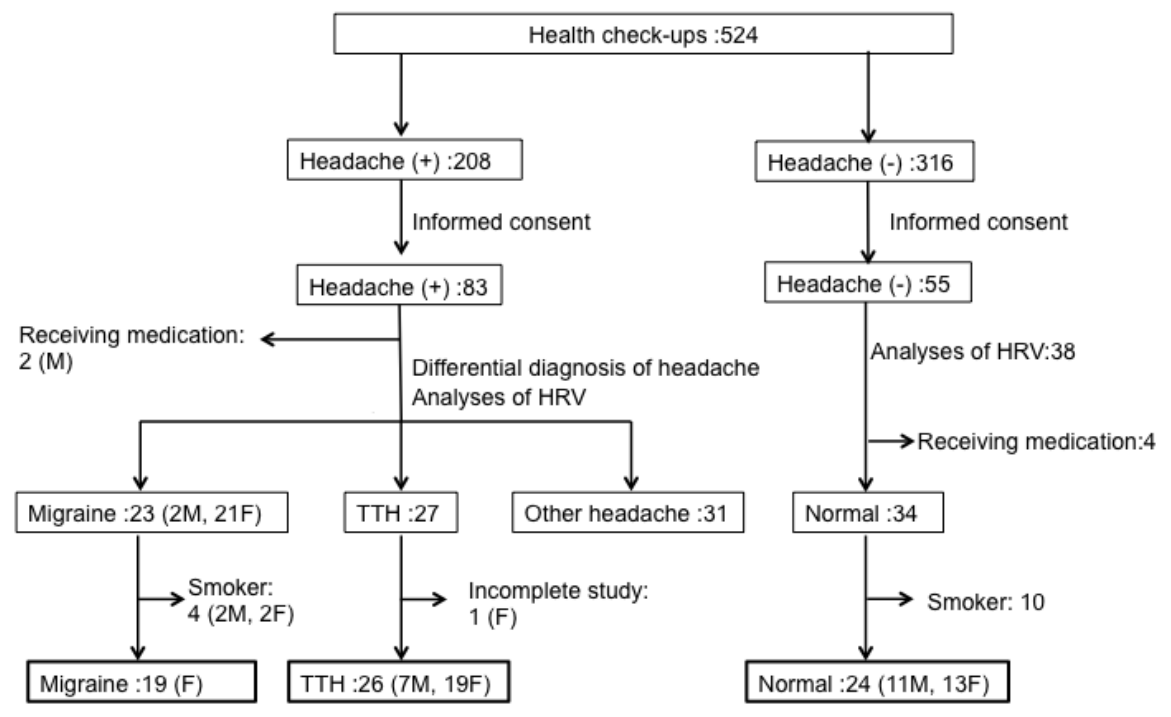

M: male, F: female

Figure 1: Study of patients underwent health check-ups at Keiju Medical Center from September 2011 to April 2013.

had experienced headache, they were asked to complete a questionnaire based on the International Classification of Headache Disorders $2^{\text {nd }}$ Edition (ICHD II). All patients who intended to participate in this study underwent an examination involving spectral analysis of HRV using APG [12]. We excluded patients who had diseases or treatments affecting autonomic functions. For instance, we excluded cases of Basedow's disease, bronchial asthma and diabetes mellitus (hemoglobin Alc $\geq 6.5 \%)$. We also excluded patients who had received medicines for diabetes mellitus, and calcium channel blockers, theophylline, corticosteroid, or alpha- or beta-blockers. Smokers were not included in this study because nicotine stimulates the activity of sympathetic nerves.

\section{Analyses of HRV using APG}

We used Artett" (U-Medica, Osaka, Japan) to measure the APG and to analyze HRV. The studies were performed at a stable temperature and under quiet conditions in morning without taking breakfast. Patients sat on chairs and put their arms on a table at a position at the same level as their heart. Patients were asked to keep quiet for 5 minutes, and then asked to put their index finger into the sensor. After collection of data in a sitting position for 2 minutes, data with an orthostatic load were collected for 2 minutes. The method of measurement was the similar to our previous report [8]. The acceleration of pulse wave was calculated by software. For spectral analyses, we utilized the maximam entropy method. We set a low-frequency (LF) range from 0.04 to $0.15 \mathrm{~Hz}$ and a high-frequency (HF) range from 0.15 to $0.40 \mathrm{~Hz}$. The densities of the power spectrum were calculated from LF and HF, respectively, and then the power ratio of LF/HF was obtained, which was estimated for the sympathetic activities. As an index of parasympathetic activities, we used the power spectrum of HF data. We also estimated the coefficient of variation of A-A intervals (CVa-a, \%) as an index of parasympathetic activities, which corresponded to CVr-r of ECG.

\section{Questionnaire}

To evaluate patients' mental and sleep statuses, we obtained data using the General Health Questionnaire-28 (GHQ-28) [13], Epworth
Sleepiness Scale (ESS) [14] and Athens Insomnia Scale (AIS) [15]. To evaluate the severities of migraine, we utilized the Japanese version of the Migraine Disability Assessment (MIDAS) [16].

\section{Statistical analysis}

To test the statistical significance of the parameters, we used analysis of variance (ANOVA) to test the homogeneity of variance of the samples. In the case of the homogeneity of variance in the samples, we performed Tukey's HSD analysis. If the samples did not exhibit homogeneity of variance, we performed Kruskal-Wallis analysis of the independent samples. To test the correlation between the MIDAS scores and markers of HRV spectroanalyses, we calculated Pearson correlation coefficients. The level of significance was set at less than $5 \%$. We used SPSS Ver. 19 (IBM Inc., USA) for statistical analyses. The cutoff values of indexes were obtained as the values for migraine and TTH with ROC curves.

\section{Results}

\section{Characteristics of patients}

In total, informed consents were obtained from 138 cases (headache $(+)$; 83 , headache $(-) ; 55)$ in this study. There were 83 patients with headache having no diseases affecting their autonomic functions. These patients neither were not received regular treatments for their headaches nor received regular medication affecting their autonomic functions. According to the criteria of ICHD II, we obtained the data of 23 patients with migraine and 27 patients with TTH. We also obtained the data of 34 age-matched healthy controls without headache. From these patients, we excluded smokers. One patient from TTH was excluded because of incomplete study. Finally, we used the data from 19 migraine patients (male 0 , female 19), 26 with TTH (male 7, female 19) and 24 normal controls (male 11, female 13) (Table 1). The process of patient selection is shown in Figure 1.

In terms of the genders of the patients, female predominance was shown in both migraine and TTH groups. The average age of patients with migraine was $48.1 \pm 8.11$ years old (mean $\pm \mathrm{SD}$ ) and that of patients 
Citation: Edahiro S, Maruta T, Negami M, Adachi Y, Yoshikawa H (2015) Spectral Analyses of Heart Rate Variability by Acceleration Plethysmography for Diagnostic Support of Migraine: Clinical Research. J Neurol Disord 3: 229. doi: 10.4172/2329-6895.1000229

Page 3 of 4

with TTH was $49.9 \pm 8.54$ years old (mean \pm SD), with no significant difference between them. There were 3 patients suffering from migraine with aura. There were no differences in age and body mass index (BMI) between the migraine and TTH groups (Table 1). The mean score of MIDAS was $4.32 \pm 5.52$ (mean \pm SD) in patients with migraine.

\section{Spectral power analyses of HRV}

We performed spectral power analyses of HRV using the data of APG (Table 2). Although the LF power in a standing position in the migraine group was lower than that in the normal controls ( $\mathrm{p}=0.0237$ ), there was no difference between the LF powers in standing position of the migraine group and TTH group. The HF power ratio of standing/sitting in the migraine group was higher than those in the TTH $(\mathrm{p}=0.0277)$ and normal groups $(\mathrm{p}=0.0104)$. The cut-off value of 1.058 could separate migraine from TTH cases with sensitivity of 0.632 and specificity of 0.846 . Furthermore, the LF/HF power ratio of standing/sitting in the migraine group was lower than those in the TTH $(p=0.0072)$ and those in the normal groups $(p=0.0102)$. The MIDAS score did not significantly correlate with the LF/HF power in

\begin{tabular}{|l|c|c|c|}
\hline & Migraine & TTH & Normal \\
\hline Gender (male/female) & $0 / 19$ & $7 / 19$ & $11 / 13$ \\
\hline Age $($ years $)$ & $48.1 \pm 8.11$ & $49.9 \pm 8.54$ & $49.1 \pm 10.1$ \\
\hline BMI $\left(\mathrm{kg} / \mathrm{m}^{2}\right)$ & $22.5 \pm 3.10$ & $23.9 \pm 3.63$ & $23.3 \pm 2.44$ \\
\hline MIDAS score & $4.32 \pm 5.52$ & & \\
\hline
\end{tabular}

$($ mean $\pm S D)$

TTH: Tension-Type Headache

BMI: Body Mass Index

Table 1: Patients

\begin{tabular}{|c|c|c|c|}
\hline & $\begin{array}{c}\text { Migraine } \\
(n=19)\end{array}$ & TTH $(n=26)$ & $\begin{array}{c}\text { Normal } \\
(n=24)\end{array}$ \\
\hline Parameter & mean \pm SD & mean \pm SD & mean \pm SD \\
\hline Heart rate in sitting position (bpm) & $74.5 \pm 14.0$ & $68.4 \pm 7.25$ & $69.5 \pm 8.54$ \\
\hline Heart rate in standing position (bpm) & $81.2 \pm 9.78$ & $74.3 \pm 8.66$ & $76.0 \pm 10.3$ \\
\hline Heart rate of standing/sitting ratio & $1.10 \pm 0.11$ & $1.09 \pm 0.09$ & $1.09 \pm 0.09$ \\
\hline Cva-a in sitting position (\%) & $5.60 \pm 5.41$ & $3.85 \pm 1.14$ & $3.72 \pm 1.47$ \\
\hline Cva-a in standing position (\%) & $3.68 \pm 1.45$ & $3.81 \pm 1.14$ & $3.70 \pm 1.52$ \\
\hline Cva-a of standing/sitting ratio & $0.83 \pm 0.31$ & $1.01 \pm 0.26$ & $1.03 \pm 0.35$ \\
\hline LF power in sitting position (\%) & $30.1 \pm 15.1$ & $30.7 \pm 16.5$ & $41.4 \pm 16.7$ \\
\hline LF power in standing position (\%) & $25.9 \pm 14.1$ & $27.6 \pm 14.3$ & $36.8 \pm 15.7$ \\
\hline LF power of standing/sitting ratio & $0.93 \pm 0.18$ & $1.19 \pm 0.15$ & $1.15 \pm 0.16$ \\
\hline HF power in sitting position (\%) & $33.2 \pm 50.5$ & $40.8 \pm 51.1$ & $27.3 \pm 16.0$ \\
\hline HF power in standing position (\%) & $28.5 \pm 18.2$ & $23.4 \pm 26.1$ & $18.1 \pm 14.0$ \\
\hline HF power of standing/sitting ratio & $1.33 \pm 0.93$ & $0.76 \pm 0.67$ & $0.80 \pm 0.69$ \\
\hline LF/HF power ratio in sitting position (\%) & $1.92 \pm 1.29$ & $2.20 \pm 2.30$ & $2.19 \pm 1.66$ \\
\hline LF/HF power ratio in standing position (\%) & $2.31 \pm 4.09$ & $2.88 \pm 2.63$ & $3.48 \pm 3.15$ \\
\hline LF/HF power ratio of standing/sitting & $0.94 \pm 0.86$ & $2.20 \pm 2.08$ & $2.28 \pm 1.93$ \\
\hline GHQA & $2.22 \pm 2.28$ & $2.65 \pm 1.69$ & $1.67 \pm 1.81$ \\
\hline GHQ B & $2.33 \pm 1.80$ & $2.20 \pm 1.82$ & $1.89 \pm 1.84$ \\
\hline GHQ C & $1.33 \pm 1.41$ & $0.45 \pm 0.83$ & $0.72 \pm 2.11$ \\
\hline GHQ D & $0.44 \pm 0.53$ & $0.30 \pm 1.13$ & $0.22 \pm 0.73$ \\
\hline GHQ Total & $6.33 \pm 4.24$ & $5.33 \pm 4.27$ & $4.50 \pm 5.51$ \\
\hline AIS & $2.96 \pm 2.01$ & $4.90 \pm 3.87$ & $3.30 \pm 2.85$ \\
\hline ESS & $8.64 \pm 3.04$ & $9.29 \pm 3.13$ & $6.88 \pm 4.20$ \\
\hline
\end{tabular}

Cva-a: A-A intervals, LF: low frequency, HF: high frequency

GHQ: General Health Questionnaire, AIS: Athens Insomnia Scale, ESS: Epworth Sleepiness Scale.

Table 2: Measurements of subjects. a standing-position/sitting-position in the migraine group. In terms of the results of the questionnaire survey, there was no difference among the groups (Table 2).

\section{Discussion}

Studies have investigated the pathophysiological mechanisms of headache, focusing on the autonomic functions [5,17]. These evaluations of autonomic functions utilized data on blood pressure or pulse rate. They also tested these data in the head-up tilt test or Valsalva test. Recently, spectral analyses of HRV were introduced in many areas $[10,18,19]$. It is supposed that the HF component of HRV reflects parasympathetic activity and the LF component represents sympathetic and parasympathetic activities [20]. In many studies, the LF/HF power ratio has been utilized as an index of sympathovagal balance $[8,20]$. Autonomic activities are usually influenced by the body's position. In a supine or sitting position, parasympathetic activity is dominant. In contrast, sympathetic activity surpasses parasympathetic activity upon being subjected to an orthostatic load.

We studied whether there were any differences in spectral power of HRV in patients with migraine, TTH, and normal control. We focused on the phenomenon that orthostatic load increased sympathetic activities [19], and attempted to evaluate dynamic autonomic responses in patients. We showed that the HF ratio of standing/sitting position in migraine patients were significantly larger than those of TTH or normal control. It suggested that the parasympathetic activity of patients with migraine increased by orthostatic loads but it decreased in patients with TTH and normal controls (Table 2). We also noticed that an orthostatic load increased the ratio of LF/HF in TTH and normal controls, but it did not cause alterations in patients with migraine. These observations might be brought by the HF elevation by orthostatic load in patients with migraine. The elevation of parasympathetic activity by orthostatic load is unusual phenomenon in normal controls. Besides, no alterations of sympathetic activity (LF/HF) followed by an orthostatic load in patients with migraine is a unique finding compared to TTH and normal control (Table 2). This finding is compatible with a report by Gotoh et al., studied by other methods [4]. They tested patients with migraine using the head-up tilt test and the Valsalva test, and found that sympathetic activities were decreased because the blood pressure declined in patients. Barloese et al., studied patients with cluster headache with the head-up tilt table test and recorded the LF, HF and LF/HF. They found decreased response of these indexes by the head-up tilt in patients with cluster headache [21]. We collected the data of LF, HF and LF/HF, and found HF ratio by orthostatic load as a supportive diagnostic measure for migraine. Because diagnoses of headache according to ICHD-II alone might have some difficulties in daily clinical settings [22], our method will be a helpful tool for differential diagnoses. According to Task Force of the European Society of Cardiology and the North American Society of Pacing and Eliectophysiology, the data collection required at least two minutes to obtain reliable results [23]. We followed this guideline and collected data.

In the course of spectral analyses of HRV, the factors affecting autonomic nervous function should be excluded. We designed our study using health check-ups. We carefully excluded diseases and condition affecting patients' autonomic functions. Our patients with headache did not receive regular medication for their headaches. In our study, there were no male patients with migraine, so the influence of gender was unclear. An influence of age was not observed in both migraine and TTH cases. Stress, anxiety, sleeplessness and fatigue are also factors affecting autonomic tone [9,11]. Therefore, we focused on these factors, and the conditions of testing were kept uniform. 
Citation: Edahiro S, Maruta T, Negami M, Adachi Y, Yoshikawa H (2015) Spectral Analyses of Heart Rate Variability by Acceleration Plethysmography for Diagnostic Support of Migraine: Clinical Research. J Neurol Disord 3: 229. doi: 10.4172/2329-6895.1000229

It is well known that migraine patients experience difficulties in the activities of daily living and social activities [24,25]. However, we could find no difference in the scores of GHQ-28, AIS and ESS. It is known that patients with TTH have sleep disturbances [26,27]. Additionally, there was no correlation between the indexes of HRV and GHQ-28, ESS points or MIDAS scores. Further studies with a larger number of patients should be considered.

\section{Conclusion}

The current study, to our knowledge, provided the first objective diagnostic method of migraine. The elevation of the HF ratio (standing/sitting) might be a supportive tool for differential diagnosis of migraine from TTH and normal controls. The method is easy to perform at the daily clinical settings.

\section{Acknowledgement}

The work was supported by Japan Society for the Promotion of Science (JSPS) Grants-in-Aid for Scientific Research <KAKENHI> Grant Number 24591253.

We thank Prof. M. Yamada and Associate Prof. K. Iwasa of the Department of Neurology and Neurobiology of Aging, Kanazawa University Graduate Schoo of Medical Sciences, and Dr. M. Kanno, Chief Director of Keiju Medical Center, for their kind support in this work.

\section{References}

1. Silberstein SD (2004) Migraine pathophysiology and its clinical implications. Cephalalgia 24 Suppl 2: 2-7.

2. Pietrobon D, Moskowitz MA (2013) Pathophysiology of migraine. Annu Rev Physiol 75: 365-391.

3. Levy D, Strassman AM, Burstein R (2009) A critical view on the role of migraine triggers in the genesis of migraine pain. Headache 49: 953-957.

4. Gotoh F, Komatsumoto S, Araki N, Gomi S (1984) Noradrenergic nervous activity in migraine. Arch Neurol 41: 951-955.

5. Peroutka SJ (2004) Migraine: a chronic sympathetic nervous system disorder Headache 44: 53-64.

6. Gass JJ, Glaros AG (2013) Autonomic dysregulation in headache patients. Appl Psychophysiol Biofeedback 38: 257-263.

7. Yerdelen D, Acil T, Goksel B, KarataÅŸ M (2007) Autonomic function in tensiontype headache. Acta Neurol Belg 107: 108-111.

8. Negami M, Maruta T, Takeda C, Adachi Y, Yoshikawa H (2013) Sympathetic skin response and heart rate variability as diagnostic tools for the differential diagnosis of Lewy body dementia and Alzheimer's disease: a diagnostic test study. BMJ Open 3.

9. Tanaka M, Mizuno K, Yamaguti K, Kuratsune H, Fujii A, et al. (2011) Autonomic nervous alterations associated with daily level of fatigue. Behav Brain Funct 7: 46 .

10. Asahina M, Mathias CJ, Katagiri A, Low DA, Vichayanrat E, et al. (2014) Sudomotor and cardiovascular dysfunction in patients with early untreated Parkinson's disease. J Parkinsons Dis 4: 385-393.

11. Shinba T, Kariya N, Matsui Y, Ozawa N, Matsuda Y, et al. (2008) Decrease in heart rate variability response to task is related to anxiety and depressiveness in normal subjects. Psychiatry Clin Neurosci 62: 603-609.
12. Takada M, Ebara T, Sakai Y (2008) The Acceleration Plethysmography System as a New Physiological Technology for Evaluating Autonomic Modulations. Health evaluation and promotion 35: 373-377.

13. Goldberg DP, Hillier VF (1979) A scaled version of the General Health Questionnaire. Psychol Med 9: 139-145.

14. Johns MW (1991) A new method for measuring daytime sleepiness: the Epworth sleepiness scale. Sleep 14: 540-545.

15. Okajima I, Nakajima S, Kobayashi M, Inoue Y (2013) Development and validation of the Japanese version of the Athens Insomnia Scale. Psychiatry Clin Neurosci 67: 420-425.

16. ligaya M, Sakai F, Kolodner KB, Lipton RB, Stewart WF (2003) Reliability and validity of the Japanese Migraine Disability Assessment (MIDAS) Questionnaire. Headache 43: 343-352.

17. Shechter A, Stewart WF, Silberstein SD, Lipton RB (2002) Migraine and autonomic nervous system function: a population-based, case-control study. Neurology 58: 422-427.

18. Heart rate variability: standards of measurement, physiological interpretation and clinical use. Task Force of the European Society of Cardiology and the North American Society of Pacing and Electrophysiology. (1996) Circulation.17: 354-381.

19. Palma JA, Benarroch EE (2014) Neural control of the heart: recent concepts and clinical correlations. Neurology 83: 261-271.

20. Chen SP, Yang AC, Fuh JL, Wang SJ (2013) Autonomic dysfunction in reversible cerebral vasoconstriction syndromes. J Headache Pain 14: 94.

21. Barloese M, Brinth L, Mehlsen J, Jennum P, Lundberg HI, et al. (2015) Blunted autonomic response in cluster headache patients. Cephalalgia .

22. Pacheva I, Milanov I, Ivanov I, Stefanov R (2012) Evaluation of diagnostic and prognostic value of clinical characteristics of migraine and tension type headache included in the diagnostic criteria for children and adolescents in International Classification of Headache Disorders--second edition. Int $\mathrm{J}$ Clin Pract 66: 1168-1177.

23. Takada M, Ebara T, Sakai Y, Kuwano Y (2009) Stationarity of the heart rate variability by acceleration plethysmography: short-term measurements of healthy young males in daily life. J Hum Ergol (Tokyo) 38: 41-50.

24. Solomon GD, Skobieranda FG, Gragg LA (1993) Quality of life and well-being of headache patients: measurement by the medical outcomes study instrument. Headache 33: 351-358

25. Durham CF, Alden KR, Dalton JA, Carlson J, Miller DW, et al. (1998) Quality of life and productivity in nurses reporting migraine. Headache 38: 427-435.

26. Ødegård SS, Engstrøm M, Sand T, Stovner LJ, Zwart JA, et al. (2010) Associations between sleep disturbance and primary headaches: the third Nord-Trøndelag Health Study. J Headache Pain 11: 197-206.

27. Engstrøm M, Hagen K, Bjørk MH, Stovner LJ, Gravdahl GB, et al. (2013) Sleep quality, arousal and pain thresholds in migraineurs: a blinded controlled polysomnographic study. J Headache Pain 1: 12. 